\title{
Dosimetric Comparison of IMRT, Hybrid IMRT and Hybrid VMAT For Early Stage Right-Sided Breast Cancer
}

\section{Suyan Bi}

Cancer Hospital Chinese Academy of Medical Sciences Shenzhen hospital https://orcid.org/00000001-7992-4078

\section{Rui Zhu}

yunyang Country people's Hospital Chongqing City

\section{Zhitao Dai ( $\nabla$ daizt_sinap@163.com )}

Cancer Hospital Chinese Academy of Medical Sciences Shenzhen hospital https://orcid.org/00000003-0554-689X

\section{Research}

Keywords: Dosimetric, second cancer risk, IMRT, hybrid IMRT, hybrid VMAT

Posted Date: July 22nd, 2021

DOI: https://doi.org/10.21203/rs.3.rs-665208/v1

License: (c) (1) This work is licensed under a Creative Commons Attribution 4.0 International License. Read Full License 


\title{
Dosimetric comparison of IMRT, hybrid IMRT and hybrid VMAT for early stage right-sided breast cancer
}

\author{
Suyan Bi ${ }^{1, \#}$ Rui Zhu ${ }^{2, \#}$ Zhitao Dai ${ }^{1{ }^{1, *}}$ \\ China; \\ ${ }^{2}$ Department of Oncology, Yunyang County People's Hospital, Chongqing, 404500, China ; \\ \#These authors contribute equally to the article; \\ *Corresponding Author: Zhitao Dai Email:daiztsinap@163.com
}

${ }^{I}$ National Cancer Center/National Clinical Research Center for Cancer/Cancer Hospital \& Shenzhen Hospital, Chinese Academy of Medical Sciences and Peking Union Medical Col lege, Shenzhen, 518116, China, Shenzhen, 518116,

\begin{abstract}
Purpose: This study aimed to evaluate the clinical impact of hybrid intensity-modulated radiotherapy (IMRT) and hybrid volumetric-modulated arc therapy (VMAT) for early-stage breast cancer, including plan quality and second cancer risk (SCR). Methods: Three different plans were designed in full IMRT, hybrid IMRT, and hybrid VMAT for each of eight patients with early-stage breast cancer. Target quality, organs at risk (OARs) sparing, and SCR were compared among the three plans. Results: Compared with the hybrid IMRT, full IMRT showed deterioration in terms of D2\% of simultaneous integrated boost (SIB), V10 of ipsilateral lung, and excess absolute risk (EAR) to contralateral lung and esophagus. The homogeneity index (HI) of SIB, V5 of ipsilateral lung and combined lung, the Dmax and Dmean of the esophagus, the EAR to contralateral breast and lung, and the EAR to the esophagus with hybrid VMAT dramatically increased by $12.5 \%, 19.49 \%, 18.87 \%, 90.59 \%, 167.69 \%$, $50.14 \%, 264.68 \%$, and $160.95 \%$, respectively $(\mathrm{p}=0.022 ; 0.040 ; 0.044 ; 0.041 ; 0.003 ; 0.020 ; 0.000$; 0.003). The EAR to contralateral breast and contralateral lung by full IMRT was significantly decreased compared with the hybrid VMAT $(26.97 \%, p=0.033 ; 50.01 \%, p=0.026)$. Conclusion: The results confirmed that hybrid IMRT could achieve better target quality and OARs sparing than full IMRT and hybrid VMAT for early-stage right breast cancer. Hybrid IMRT was the best treatment option, while hybrid VMAT performed the worst among the three plans in terms of SCR to peripheral OARs.
\end{abstract}

Keywords: Dosimetric; second cancer risk; IMRT; hybrid IMRT; hybrid VMAT 


\section{Introduction}

Usually diagnosed as early-stage female cancer, the 5-year specific survival rate of breast cancer is up to $98.9 \%$ [1]. Whole breast radiotherapy (RT) and a boost to the tumor bed are considered as the adjuvant therapy after breast-conserving surgery for early-stage breast cancer [2,3]. Studies confirmed that patients benefited from RT and tumor bed boosting [3,4].

Various RT techniques, such as three-dimensional conformal radiation therapy (3D-CRT), intensity-modulated radiation therapy (IMRT), and volumetric-modulated arc therapy (VMAT), have been adopted for treating breast cancer. Utilizing two opposed, wedged, and tangential fields, 3D-CRT treating the whole breast is carried out with multi-leaf collimators (MLCs) to shield the adjacent normal tissue. 3D-CRT has the advantage of improving the local control, but the toxicities associated with radiation to the organs at risk (OARs) are a concern [5]. Dividing each treatment beam into smaller beam segments, IMRT delivers a non-uniform fluence to optimize the dose distribution [5]. VMAT can rotate the angle of gantry and radiate beams continuously, and modulate the dose rate (DR) and the shape of the MLCs simultaneously to achieve a highly conformal dose coverage [6]. IMRT and VMAT were reported to have incomparable advantages in dose homogeneity and coverage compared with 3D-CRT [6,7]. However, IMRT might be more susceptible to setup error and shape changes of the breast in whole breast RT [8]. To reduce the effects of the geometrical uncertainties, Nakamura et al. [8] proposed a method of hybrid IMRT plan comprised of two opposed tangential open beams and two inverse-planned IMRT beams. And they proved the hybrid IMRT had excellent performance in target quality and offsetting the geometrical uncertainties for patients who underwent whole breast RT [8].

RT resulted in inevitably radiation damage and therapy-related second cancer risk (SCR) for normal tissue, which was confirmed by studies $[9,10]$. With the improvement of the efficacy and overall survival of breast cancer patients, the SCR and radiation toxicity caused by RT has gradually become a research focus. Early studies showed that 3D-CRT possesses a lower SCR than IMRT and VMAT [11,12].

To pursue an excellent target dose coverage and OARs sparing, and also lower the SCR and radiation toxicity, selecting a reasonable RT modality is critical for treating breast cancer. To the best of our knowledge, the clinical impact of hybrid VMAT for breast cancer have not been studied. This study aims to assess the plan quality and SCR among three treatment modalities (full IMRT, hybrid IMRT, and hybrid VMAT) for breast cancer.

\section{Materials and methods}

\section{Patients preparation}

Eight females aged between 41 and 51 years old, with early-stage right-sided breast cancer after breast-conserving surgery, were randomly selected. None of the patients had contraindications for RT. This study was approved by the ethics committee of Chongqing university Cancer Hospital, and the informed consent was acquired from each enrolled patient.

All of the patients were positioned with a breast bracket and fixed foam plate on the affected side of the lower limbs. The computed tomography (CT) scans were acquired on a Philips Brilliance Big 
Bore CT (Philips, Holland) simulation in 5-mm-thick slices, in the supine position with the scan scope from the mandible to the thorax. In addition, all of the adjacent normal tissues, such as the heart, lung, esophagus, and contralateral breast, were completely covered.

\section{Contouring of target volumes and OARs}

Target volumes and OARs were delineated on the Eclipse treatment planning system (TPS, Varian Medical Systems, Version 13.6, Inc.). The clinical target volume (CTV) and the boost region were delineated by the same radiation oncologist on each CT dataset. The CTV was the whole breast tissue identifiable on the CT scan assisted by wire markers, which were placed around the palpable breast tissue during the simulation. Then the CTV limited posteriorly by the intercostal front and retracted $5 \mathrm{~mm}$ from the skin. The boost region encompassed the surgical bed or seroma. The planning target volume (PTV) was expanded $5 \mathrm{~mm}$ based on the CTV, excluding the heart. Then the PTV was retracted $5 \mathrm{~mm}$ from the skin and limited posteriorly by the intercostal front. The boost region was expanded by $5 \mathrm{~mm}$ in all directions to create the SIB (simultaneous integrated boost) volume. The contoured OARs were the contralateral breast, heart, spinal cord, esophagus, and ipsilateral and contralateral lungs.

\section{RT plans}

Figure 1 and Figure 2 show the fields distributions and the dose distributions in CT images for the three RT techniques respectively.Three different RT plans (full IMRT, hybrid IMRT, and hybrid VMAT) were created for each case in the Eclipse TPS. Utilizing $6 \mathrm{MV}$ photon beams generated by Varian IX linear accelerator, dose optimization and calculations were done in Eclipse TPS for all of the plans. The algorithms of Dose-Volume Optimizer and Progressive Resolution Optimizer were used for IMRT, and VMAT dose optimizations, respectively, and Anisotropic Analytical Algorithm was adopted for final dose calculations [13, 14].

\section{1) Full IMRT}

The full IMRT plans contained two opposed tangential fields, and another four fields, which were at the angles of $10^{\circ}$ or $20^{\circ}$ to the two tangential fields in the direction of outside the body. The angles of the collimator and the position of jaws of all of the fields were adjusted before dose optimization to maximize the protection of the lungs. All of the fields were delivered with a dynamic sliding-window IMRT delivery technique and the fixed DR of 400 monitor units (MUs) $/ \mathrm{min}$.

\section{2) Hybrid IMRT}

The hybrid IMRT plans owned two opposed tangential open beams plus three IMRT beams. Two of the three IMRT beams were at the angles of $10^{\circ}$ to the two tangential fields in the direction of outside the body, and the third IMRT beam had an angle of about $30^{\circ}$ to $45^{\circ}$ to the tangential field on the upper side avoiding exposure to the heart and contralateral breast. To maximize the protection of the lungs, the angles of the collimator of the three IMRT beams were adjusted, and the position of the jaws of the third IMRT beam was adjusted and fixed, adapting the shape of the SIB before dose optimization and calculation. The adopted delivery technique and DR were the same as that of the full 
IMRT plans. The open beams contributed $80 \%$ of the total dose, whereas the inversely optimized IMRT beams contributed to the remaining prescription dose.

\section{3) Hybrid VMAT}

The hybrid VMAT plans owned two opposed tangential open beams and a half arc beam. The gantry of the arc beam rotated from one tangential angle to the other tangential angle. The maximum DR of the arc beam was set to $600 \mathrm{MUs} / \mathrm{min}$. The open beams contributed with $80 \%$ of the total dose, whereas the inversely optimized arc beams contributed to the remaining prescribed dose.

For the SIB and PTV-SIB of all of the plans, the prescribed doses were 50 and 45 Gy in 25 fractions, respectively. The prescribed $95 \%$ isodose covered no less than $95 \%$ of the target volume [15], and the percentage volume of the target volume radiated over $110 \%$ of the prescribed dose was no more than $2 \%$. The dose constraints for adjacent OARs of contralateral breast, heart, ipsilateral lung, contralateral lung, spinal cord, and esophagus were defined according to published literature [5].

\section{Treatment plan evaluation}

The data collected from the Dose-Volume Histogram (DVH) of all of the plans were evaluated in the aspect of target coverage and OARs sparing. Figure 3 shows the representative DVHs for the three RT techniques.

SIB: the maximum dose $\left(D_{\max }\right)$, the mean dose $\left(D_{\text {mean }}\right)$, and $V_{95 \%}$ of SIB were assessed. The $D_{\max }$ of SIB, also named $\mathrm{D}_{2} \%$, is defined as the dose received by $2 \%$ of the target volume, and $\mathrm{V}_{95 \%}$ is defined as the percentage volume of the target volume receiving $95 \%$ of the prescribed dose. The conformal index (CI) and homogeneity index (HI) were also evaluated. The CI of SIB is defined as $\mathrm{CI}=\mathrm{TV}_{\mathrm{PTV}}^{2} /(\mathrm{TV} \times \mathrm{PIV})$ utilizing the Paddick conformity index, where the $\mathrm{TV}_{\mathrm{PTV}}$ was the SIB volume receiving $95 \%$ of the prescription dose, the TV is the total volume of the SIB, and the PIV is the total volume covered by the prescribed $95 \%$ isodose. The HI of SIB was assessed using HI= $\left(\mathrm{D}_{5 \%}-\mathrm{D}_{95 \%}\right) / \mathrm{D}_{\text {mean }}$, where $\mathrm{D}_{5 \%}$ and $\mathrm{D}_{95 \%}$ are the minimum dose radiated to $5 \%$ and $95 \%$ of the SIB, respectively.

PTV-SIB: the $\mathrm{D}_{2 \%}$, the $\mathrm{D}_{\text {mean }}, \mathrm{V}_{95} \%$, and CI of PTV-SIB were assessed. These indicators were defined as described above.

OARs: the $\mathrm{D}_{\max }$ and $\mathrm{D}_{\text {mean }}$ of contralateral breast, Heart, spinal cord and esophagus, and the $\mathrm{D}_{\text {mean }}$ of contralateral lung were executed for dosimetric analysis. The $\mathrm{V}_{5}$ (the percentage volume receiving 5 $\mathrm{Gy}$ ), $\mathrm{V}_{10}$ (the percentage volume receiving $10 \mathrm{~Gy}$ ), $\mathrm{V}_{20}$ (the percentage volume receiving $20 \mathrm{~Gy}$ ), $\mathrm{V}_{30}$ (the percentage volume receiving $30 \mathrm{~Gy}$ ), and $\mathrm{D}_{\text {mean }}$ of the ipsilateral lung and combined lung were also evaluated.

\section{SCR calculations}

The SCR caused by RT of normal tissues can be assessed by Model excess absolute risk (EAR), as proposed by Schneider [16,17]. The Equation (1) shown below can be utilized to calculate the SCR of an organ [18,19]:

$$
E A R^{\text {org }}=\frac{1}{V_{T}} \sum_{i} V\left(D_{i}\right) \beta_{E A R R E D\left(D_{i}\right) \mu(x, a)}
$$


where $V_{T}$ is the total organ volume assessed for secondary carcinogenesis, $V\left(D_{i}\right)$ represents the organ volume receiving the dose $D_{i}$, and the parameter $\beta_{E A R}$ is the slope of the dose-response curve in the low dose region. Equation (2), $R E D\left(D_{i}\right)$, represents the dose-response mechanistic model, which describes the fractionation effects and cell killing:

$$
R E D\left(D_{i}\right)=\frac{e^{-\alpha^{\prime} D i}}{\alpha^{\prime} R}\left(1-2 R+R^{2} e^{\alpha^{\prime} D i}-(1-R)^{2} e^{-\frac{\alpha^{\prime} R}{1-R} D i}\right)
$$

where $R$ is a parameter that represents the repopulation or repair ability of normal tissues between two dose fractions, and the parameter $\alpha^{\prime}$ was calculated by Equation (3):

$$
\alpha^{\prime}=\alpha+\beta d=\alpha+\beta D_{i} / D_{T} d_{T}
$$

where $D_{T}$ is the prescribed dose of $50 \mathrm{~Gy}$ to the SIB in this study, and $d_{T}$ represents the corresponding fractionation dose of $2 \mathrm{~Gy}$. Given by Equation (4), $\mu(x, a)$ expresses the modifying function:

$$
\mu(x, a)=e^{[\gamma e(x-30)+\gamma a \ln (a / 70)]}
$$

where $\gamma_{e}$ and $\gamma_{a}$ are both the age modifying parameters.

In this study, the EAR has been investigated to the organs of contralateral breast, contralateral lung, ipsilateral lung, and esophagus. The assumed value of $\alpha / \beta=3$ Gy for all of the organs needed to evaluate EAR, and all of the other parameters used in EAR calculation were selected from previous research [18]. The parameters are shown in Table 1.

\section{Statistical analysis}

To determine whether the pair parameters were different, a paired t-test was carried out using the Microsoft Excel. If the $p$-value is less than 0.05 , the difference is considered to be statistically significant.

\section{Results}

\section{Target coverage}

The parameters of $\mathrm{D}_{2 \%}, \mathrm{D}_{\text {mean }}, \mathrm{V}_{95 \%}, \mathrm{CI}$, and $\mathrm{HI}$ were compared to evaluate the quality of target dose coverage. For the SIB, the hybrid IMRT obtained a lower $\mathrm{D}_{2 \%}$ than both full IMRT and hybrid VMAT ( $\mathrm{p}<0.05$ ) and achieved better HI than the hybrid VMAT ( $<<0.05$ ). For the PTV-SIB, the $\mathrm{V}_{95 \%}$ of the hybrid IMRT $(99.40 \pm 0.50)$ was better than that of the hybrid VMAT $(99.07 \pm 0.56)(\mathrm{p}<0.05)$. The findings on SIB and PTV-SIB are listed in Table 2.

\section{OARs}

The delivered doses to the OARs are listed in Table 3. Compared with the hybrid IMRT, the $\mathrm{V}_{5}$ of ipsilateral lung and combined lung with hybrid VMAT increased by $19.45 \%$ and $18.87 \%$, respectively $(\mathrm{p}=0.040 ; 0.044)$, the $\mathrm{V}_{10}$ of the ipsilateral lung with full IMRT increased $4.13 \mathrm{~Gy}(\mathrm{p}=0.012)$, and the $D_{\max }$ and $D_{\text {mean }}$ of the esophagus with hybrid VMAT dramatically increased by $90.59 \%$ and $167.69 \%$, respectively $(\mathrm{p}=0.041 ; 0.003)$. 


\section{SCR calculations}

The EAR of the organs of contralateral breast, contralateral lung, ipsilateral lung, and esophagus with three treatment modalities are shown in Table 4. Compared with hybrid VMAT, the EAR to the contralateral breast with full IMRT and hybrid IMRT were decreased by $26.97 \%$ and $33.39 \%$, respectively ( $\mathrm{p}=0.033 ; 0.020)$, and the EAR to the contralateral lung with full IMRT and hybrid IMRT were reduced by $50.01 \%$ and $72.58 \%$, respectively $(\mathrm{p}=0.026 ; 0.000)$. In comparison with the hybrid IMRT, the EAR to the esophagus with full IMRT and hybrid VMAT increased $80.21 \%$ and $160.95 \%$, respectively $(\mathrm{p}=0.028 ; 0.003)$.

\section{Discussion and conclusion}

Since studies evaluating the hybrid IMRT and hybrid VMAT for early-stage breast cancer are rare, a comparison of the target dose coverage, OARs sparing, and SCR among full IMRT, hybrid IMRT, and hybrid VMAT for treating early-stage breast cancer is extremely relevant. This study aimed to estimate the three RT plans, and the expectation was to bring more clinical options to RT for early-stage right-sided breast cancer.

IMRT showed a significant advantage in target dose coverage, and surrounding OARs spring for left-sided breast cancer after breast-conserving surgery $[5,6,20]$. This could result in better tumor control rate and lower toxicity, and late effects compared with the conventional tangential pair treatment beams. However, IMRT had inherent geometrical uncertainties arising from the setup error and target motion, which offset the merits of IMRT for breast cancer [7, 8, 21]. Combining two opposed tangential open beams and IMRT beams, the hybrid IMRT plan might solve the geometrical uncertainties of IMRT. Nakamura et al. [8] compared the plan quality and robustness of the dose distributions against setup and motion uncertainties among four RT plans. They confirmed that hybrid IMRT performed better robustness against the uncertainties than full IMRT, and it offered excellent plan quality. Fogliata et al. [22] compared the dosimetric difference for the involved OARs among 3D-CRT plan with field in field technique, and two VMAT plans (VMAT_full and VMAT_tang, gantry rotation partial arc from about 295 to $173^{\circ}$ without and with a sector of $0 \mathrm{MU}$, respectively) for breast cancer. They proved that full VMAT had an obvious weakness in radiating a higher mean dose to the nearby OARs compared with VMAT_tang. Considering the excellent characteristics of hybrid plans and the lack of studies on hybrid VMAT plan, here, we eagerly studied the clinical dosimetric characteristics and SCR of full IMRT, hybrid IMRT, and hybrid VMAT, and we found that hybrid IMRT was superior to full IMRT and hybrid VMAT in target quality, and OARs sparing for early-stage right-sided breast cancer. Adopting the VMAT_tang (partial arcs with a sector of $0 \mathrm{MU}$ ) method from Fogliata et al.'s study, instead of two opposed tangential open beams plus a complete half arc in our study, the performance of hybrid VMAT in protecting peripheral OARs might be improved. However, different from irradiating the only target PTV as in Fogliata et al.'s study, the hybrid VMAT in our study delivered a boost dose to the tumor bed, and achieved better CI and HI for both the tumor bed and the PTV. Thus, the hybrid VMAT with a complete half arc beam might be reasonable in this study. However, the half arc beam delivered only $20 \%$ of the total dose by continuous rotation $180^{\circ}$, and the dose to the surrounding OARs inevitably increased. 
As a tumor with a better therapeutic effect and longer life expectancy than most other tumors, the radiation-related risk is the most serious sequelae for breast cancer survivors, which has been confirmed by numerous epidemiological cohort studies [23]. The occurrence of secondary cancer is closely related to the tissues and organs themselves. Studies have shown that fatal secondary cancer mainly occurs in the stomach, lungs, and colon, and the thyroid has a particularly low threshold of SCR (mean dose as low as $0.05 \mathrm{~Gy}$ in children and young adults) [23, 24]. In addition, the occurrence of secondary cancer depends on the radiation dose. Secondary cancer tends to occur in volumes receiving a total dose or near volumes receiving dose from 2 to $50 \mathrm{~Gy}$ radiation [23, 25]. Several studies demonstrated that SCR dramatically increased when receiving a dose reaching a certain range in the kidney (from 1 to $15 \mathrm{~Gy}$ ), stomach and pancreas (from 1 to $45 \mathrm{~Gy}$ ), and bladder and rectum (from 1 to $60 \mathrm{~Gy}$ ). [23, 26]. In our study, seeking the least toxic radiation modality for breast cancer, we compared the SCR of three modalities for the contralateral breast, contralateral lung, ipsilateral lung, and esophagus.

Recently, Schneider proposed a calculation model, namely, the EAR model, which can be adopted for SCR calculation and evaluation utilizing DVH data from the RT plan and related radiobiological parameters $[16,19]$. The EAR model has proved its feasibility to assess the SCR for patients with nasal natural killer T-cell lymphoma and breast cancer [19, 22]. Fogliata et al. [22] applied the EAR model to compare the SCR among 3D-CRT, VMAT_full, and VMAT_tang for breast cancer. And they confirmed that VMAT_tang had advantages in reducing RT toxicity for the ipsilateral organs compared with 3D-CRT with field in field technique when they delivered the same SCR to the contralateral organs.

In this study, we also adopted the EAR model to calculate the SCR for right-sided breast cancer, and our results demonstrated that the hybrid IMRT performed best in target quality, OARs spring, and SCR to peripheral OARs. However, if the half arc had a sector of $0 \mathrm{MU}$ in hybrid VMAT, the performance of hybrid VMAT in SCR to adjacent OARs probably approached or achieved the effect of hybrid IMRT. The percentage of radiated dose and the effective dose delivery angle for the arc beam in the VMAT_tang in Fogliata's study and the hybrid VMAT in our study was quite different. This could translate into a differentiated radiation dose and SCR to the nearby healthy tissue. Of course, the results of the EAR model in predicting SCR depend on the accuracy of commercial TPS system modeling and related biological parameters.

Hybrid IMRT combined the advantages of 3D-CRT and IMRT in treating early-stage right-sided breast cancer. Hybrid IMRT was shown to have significant advantages in target dose coverage, OARs sparing, and SCR to nearby normal tissues. Hybrid IMRT is worthy of clinical application and promotion.

\author{
Abbreviations: \\ IMRT:intensity-modulated radiotherapy \\ VMAT:volumetric-modulated arc therapy \\ SCR:second cancer risk \\ OAR:organs at risk \\ SIB:simultaneous integrated boost
}


EAR:excess absolute risk

HI:homogeneity index

RT:radiotherapy

3D-CRT:3-dimensional conformal radiation therapy

MLCs:multi-leaf collimators

DR:dose rate

CT:computed tomography

TPS:eclipse treatment planning system

CTV:clinical target volume

PTV:planning target volume

MUs:monitor units

DVH:dose-volume histogram

CI:conformal index

\section{Declarations}

\section{Ethics approval and consent to participate}

The study was approved by the institutional review board of our hospital.

\section{Consent for publication}

The consents for publication of data have been obtained from patients.

\section{Availability of data and materials}

Not applicable.

\section{Conflict of Interest Statement}

The authors have no conflicts of interest to declare.

\section{Funding}

This work was supported generously by the Basic and Applied Basic Research Foundation of Guangdong Province (GrantNo. 2020A1515110335).

\section{Author Contributions}

All authors carried out the study. Suyan Bi, Rui Zhu, Zhitao Dai draft the manuscript. All authors read and approved the final manuscript.

\section{Acknowledgment}

The authors thank Prof. Xianfeng Liu for helpful discussion.

\section{References}

1 Nienke Hoekstra, Emmanuelle Fleury, Tomas Rodrigo Merino Lara, et al: Long-term risks of secondary cancer for various whole and partial breast irradiation techniques. Radiother Oncol 2018;128(3): 428-433.

2 C. Hammer, J.H. Maduro, E.J. Bantema-Joppe, et al: Radiation-induced fibrosis in the boost area after three-dimensional conformal radiotherapy with a simultaneous integrated boost technique for early-stage breast cancer: A multivariable prediction model. Radiother Oncol 2017;122(1):45-49.

3 Pearson D, Wan J, Bogue J: A novel technique for treating deep seated breast cavity boosts. Med Dosim 2019;Sep 20.

4 Chung MJ, Kim SH, Lee JH, et al: A Dosimetric Comparative Analysis of TomoDirect and Three-Dimensional Conformal Radiotherapy in Early Breast Cancer. J Breast Cancer 2015;18(1): 57-62.

5 Michalski A, Atyeo J, Cox J, Lamoury G, et al: A dosimetric comparison of 3D-CRT, IMRT, and static tomotherapy with an SIB for large and small breast volumes. Med Dosim 2014;39(2):163-8. 
6 Mo JC, Huang J, Gu WD, et al: A dosimetric comparison of double-arc volumetric arc therapy, step-shoot intensity modulated radiotherapy and 3D-CRT for left-sided breast cancer radiotherapy after breast-conserving surgery. Technol Health Care 2017;25(5): 851-858.

7 Liu H, Chen X, He Z, et al: Evaluation of 3D-CRT, IMRT and VMAT radiotherapy plans for left breast cancer based on clinical dosimetric study. Comput Med Imaging Graph 2016,54:1-5.

8 Nakamura N, Takahashi O, Kamo M, et al: Effects of geometrical uncertainties on whole breast radiotherapy: a comparison of four different techniques. J Breast Cancer 2014;17(2):157-60.

9 Deasy JO, Moiseenko V, Marks L, et al: radiotherapy dose-volume effects on salivary gland function. Int J Radiat Oncol Biol Phys 2010;76(3):58-63.

10 Toda K, Shibuya H, Hayashi K, Ayukawa F: radiation-induced cancer after radiotherapy for non-hodgkin's lymphoma of the head and neck: a retrospective study. Radiat Oncol 2009;4:21.

11 Abo-Madyan Y, Aziz MH, Aly MM, et al: Second cancer risk after 3D-CRT, IMRT and VMAT for breast cancer. Radiother Oncol 2014;110(3):471-6.

12 Corradini S, Ballhausen $\mathrm{H}$, Weingandt $\mathrm{H}$, et al: Left-sided breast cancer and risks of secondary lung cancer and ischemic heart disease: Effects of modern radiotherapy techniques. Strahlenther Onkol 2018;194(3): 196-205.

13 Mingzan Z, Tuodan Z, Zhijian C, et al: Advanced nasopharyngeal carcinoma radiotherapy with volumetric modulated arcs and the potential role of flattening filter-free beams. Radiat Oncol 2013;8:120.

14 Bragg CM, Wingate K, Conway J: Clinical implications of the anisotropic analytical algorithm for IMRT treatment planning and verification. Radiother Oncol 2008;86:276-84.

15 Peters S, et al: A treatment planning study comparing Elekta VMAT and fixed field IMRT using the varian treatment planning system eclipse. Radiat Oncol 2014;9:153.

16 Uwe Schneider: Modeling the Risk of Secondary Malignancies after Radiotherapy. Genes 2011;2:1033-1049.

17 Schneider, U: Mechanistic model of radiation-induced cancer after fractionated radiotherapy using the linear-quadratic formula. Med. Phys 2009;36:1138-1143.

18. Uwe Schneider, Marcin Sumila, Judith Robotka: Site-specific dose-response relationships for cancer induction from the combined Japanese A-bomb and Hodgkin cohorts for doses relevant to radiotherapy. Theoretical Biology and Medical Modelling 2011;8:27.

19 Xianfeng Liu, Furong Wu, Qishuai Guo, et al: Estimation of radiotherapy modalities for patients with stage I-II nasal natural killer T-Cell lymphoma. Cancer Management and Research 2019;11:7219-7229.

20 Haiyun Liu, Xinde Chen, Zhijian He, et al: Evaluation of 3D-CRT, IMRT and VMAT radiotherapy plans for left breast cancer based on clinical dosimetric study. Computerized Medical Imaging and Graphics 2016;54:1-5.

21 Anke Van Mourik, Simon Van Kranen, Suzanne Den Hollander, et al: Effects of setup errors and shape changes on breast radiotherapy. Int. J. Radiation Oncology Biol. Phys 2011;79(5):1557-1564.

22 Fogliata A, De Rose F, Franceschini D, et al: Critical Appraisal of the Risk of Secondary Cancer Induction from Breast Radiation Therapy with Volumetric Modulated Arc Therapy Relative to 3D Conformal Therapy. Int J Radiat Oncol Biol Phys 2018;100(3):785-793.

$23 \mathrm{Fu}$ Jin, Huan-Li Luo, Juan Zhou, et al: Cancer risk assessment in modern radiotherapy workflow with medical big data. Cancer Manag Res 2018;10:1665-1675.

24 Cardis E, Howe G, Ron E, et al: Cancer consequences of the Chernobyl accident: 20 years on. J Radiol Prot 2006;26(2):127-140.

25 Berrington de Gonzalez A, Gilbert E, Curtis R, et al: Second solid cancers after radiation therapy: a systematic review of the epidemiologic studies of the radiation dose-response relationship. Int $\mathrm{J}$ Radiat Oncol Biol Phys 2013;86(2):224-233.

26 Suit H, Goldberg S, Niemierko A, et al: Secondary carcinogenesis in patients treated with radiation: a review of data on radiation-induced cancers in human, non-human primate, canine and rodent subjects. Radiat Res 2007;167(1):12-42.

\section{Figure captions:}

Figure 1. Fields distributions for (a) Full IMRT, (b) hybrid IMRT and (c) Hybrid VMAT.

Figure 2. Contouring of targets and comparison of planar dose distribution for one selected case. (a), (b),(c) are planar dose distribution of Full IMRT, Hybrid IMRT and Hybrid VMAT plans, respectively.

Figure 3. The DVHs curves of the targets with prescription dose of $43.5 \mathrm{~Gy} / 15 \mathrm{~F}$ and $49.5 \mathrm{~Gy} / 15 \mathrm{~F}$ respectively (upper rows) and OARs (lower rows) for Full IMRT plan, Hybrid IMRT plan and 
Table 1. the other parameters used in EAR

\begin{tabular}{lllllll}
\hline site & $\beta_{\text {EAR }}$ & $\gamma_{e}$ & $\gamma_{a}$ & $\alpha$ & $\alpha / \beta$ & $\mathrm{R}$ \\
\hline Contralateral breast & 9.2 & -0.037 & 1.7 & 0.044 & 3 & 0.15 \\
Contralateral lung & 7.5 & 0.002 & 4.23 & 0.042 & 3 & 0.83 \\
Ipsilateral lung & 7.5 & 0.002 & 4.23 & 0.042 & 3 & 0.83 \\
Esophagus & 0.58 & -0.002 & 1.9 & 0.026 & 3 & 0.81 \\
\hline
\end{tabular}

Table 2. The findings on SIB and PTV-SIB

\begin{tabular}{|c|c|c|c|c|c|c|c|}
\hline & \multirow[b]{2}{*}{ Parameters } & \multirow[b]{2}{*}{ full IMRT } & \multirow[b]{2}{*}{ hybrid IMRT } & \multirow[b]{2}{*}{ hybrid VMAT } & \multicolumn{3}{|c|}{$p$-Value } \\
\hline & & & & & $\begin{array}{l}\text { full IMRT vs. } \\
\text { hybrid IMRT }\end{array}$ & $\begin{array}{l}\text { full IMRT vs. } \\
\text { hybrid VMAT }\end{array}$ & $\begin{array}{l}\text { hybrid IMRT vs. } \\
\text { hybrid VMAT }\end{array}$ \\
\hline \multirow[t]{5}{*}{ SIB } & $\mathrm{D}_{2 \%}(\mathrm{~Gy})$ & $52.76 \pm 0.59$ & $52.35 \pm 0.61$ & $52.70 \pm 0.48$ & 0.044 & 0.745 & 0.046 \\
\hline & $\mathrm{D}_{\text {mean }}(\mathrm{Gy})$ & $51.20 \pm 0.43$ & $51.18 \pm 0.59$ & $51.41 \pm 0.38$ & 0.953 & 0.183 & 0.197 \\
\hline & $\mathrm{V}_{95 \%}(\%)$ & $99.88 \pm 0.34$ & $99.96 \pm 0.11$ & $100.00 \pm 0.00$ & 0.351 & 0.351 & 0.351 \\
\hline & $\mathrm{CI}$ & $0.794 \pm 0.058$ & $0.820 \pm 0.048$ & $0.821 \pm 0.046$ & 0.153 & 0.070 & 0.908 \\
\hline & HI & $0.051 \pm 0.013$ & $0.048 \pm 0.012$ & $0.054 \pm 0.010$ & 0.502 & 0.423 & 0.022 \\
\hline \multirow[t]{4}{*}{ PTV-SIB } & $\mathrm{D}_{2 \%}(\mathrm{~Gy})$ & $49.42 \pm 0.66$ & $49.32 \pm 0.34$ & $49.21 \pm 0.36$ & 0.655 & 0.318 & 0.355 \\
\hline & $\mathrm{D}_{\text {mean }}(\mathrm{Gy})$ & $45.74 \pm 0.38$ & $45.65 \pm 0.16$ & $45.60 \pm 0.26$ & 0.518 & 0.160 & 0.599 \\
\hline & $\mathrm{V}_{95 \%}(\%)$ & $98.98 \pm 0.41$ & $99.40 \pm 0.50$ & $99.07 \pm 0.56$ & 0.098 & 0.637 & 0.046 \\
\hline & CI & $0.632 \pm 0.073$ & $0.656 \pm 0.089$ & $0.650 \pm 0.09$ & 0.459 & 0.588 & 0.628 \\
\hline
\end{tabular}


Table 3. The delivered doses to the OARs

$p$-Value

Parameters full IMRT hybrid IMRT hybrid VMAT full IMRT vs. full IMRT vs. hybrid IMRT vs. hybrid IMRT hybrid VMAT hybrid VMAT

\begin{tabular}{|c|c|c|c|c|c|c|c|}
\hline \multirow[t]{2}{*}{ Contralateral breast } & $\mathrm{D}_{\max }(\mathrm{Gy})$ & $12.47 \pm 9.58$ & $12.34 \pm 13.2$ & $11.84 \pm 12.86$ & 0.933 & 0.651 & 0.113 \\
\hline & $\mathrm{D}_{\text {mean }}(\mathrm{Gy})$ & $0.82 \pm 0.55$ & $0.83 \pm 0.51$ & $1.00 \pm 0.49$ & 0.950 & 0.128 & 0.432 \\
\hline \multirow[t]{2}{*}{ Spinal cord } & $\mathrm{D}_{\max }(\mathrm{Gy})$ & $2.88 \pm 3.38$ & $2.10 \pm 1.86$ & $2.54 \pm 0.76$ & 0.258 & 0.744 & 0.417 \\
\hline & $\mathrm{D}_{\text {mean }}(\mathrm{Gy})$ & $0.63 \pm 0.53$ & $0.58 \pm 0.19$ & $0.71 \pm 0.24$ & 0.801 & 0.615 & 0.137 \\
\hline \multirow[t]{5}{*}{ Ipsilateral lung } & $\mathrm{V}_{5 \mathrm{~Gy}}(\%)$ & $33.22 \pm 23.14$ & $35.96 \pm 4.49$ & $42.97 \pm 9.41$ & 0.732 & 0.180 & 0.040 \\
\hline & $\mathrm{V}_{10 \mathrm{~Gy}}(\%)$ & $24.89 \pm 6.26$ & $20.76 \pm 3.29$ & $19.76 \pm 5.31$ & 0.012 & 0.077 & 0.607 \\
\hline & $\mathrm{V}_{20 \mathrm{~Gy}}(\%)$ & $14.42 \pm 3.41$ & $13.38 \pm 2.94$ & $13.17 \pm 3.02$ & 0.181 & 0.072 & 0.267 \\
\hline & $\mathrm{V}_{30 \mathrm{~Gy}}(\%)$ & $10.02 \pm 2.95$ & $10.48 \pm 2.79$ & $10.39 \pm 2.97$ & 0.219 & 0.230 & 0.652 \\
\hline & $\mathrm{D}_{\text {mean }}(\mathrm{Gy})$ & $18.72 \pm 13.43$ & $8.45 \pm 1.27$ & $9.01 \pm 1.73$ & 0.065 & 0.081 & 0.079 \\
\hline Contralateral lung & $\mathrm{D}_{\text {mean }}(\mathrm{Gy})$ & $0.37 \pm 0.42$ & $0.33 \pm 0.30$ & $0.74 \pm 0.34$ & 0.826 & 0.157 & 0.078 \\
\hline \multirow[t]{5}{*}{ Combined lung } & $\mathrm{V}_{5 \mathrm{~Gy}}(\%)$ & $24.09 \pm 7.87$ & $20.19 \pm 3.16$ & $24.00 \pm 5.26$ & 0.124 & 0.960 & 0.044 \\
\hline & $\mathrm{V}_{10 \mathrm{~Gy}}(\%)$ & $15.06 \pm 5.30$ & $12.37 \pm 2.83$ & $11.97 \pm 2.51$ & 0.097 & 0.142 & 0.592 \\
\hline & $\mathrm{V}_{20 \mathrm{~Gy}}(\%)$ & $9.31 \pm 1.85$ & $8.03 \pm 1.59$ & $9.48 \pm 3.54$ & 0.211 & 0.908 & 0.232 \\
\hline & $\mathrm{V}_{30 \mathrm{G}}(\%)$ & $6.42 \pm 1.31$ & $6.27 \pm 1.41$ & $7.45 \pm 3.18$ & 0.793 & 0.361 & 0.244 \\
\hline & $\mathrm{D}_{\text {mean }}(\mathrm{Gy})$ & $4.54 \pm 2.20$ & $3.38 \pm 2.60$ & $5.08 \pm 1.82$ & 0.282 & 0.545 & 0.259 \\
\hline \multirow[t]{2}{*}{ Esophagus } & $\mathrm{D}_{\max }(\mathrm{Gy})$ & $3.39 \pm 3.85$ & $2.02 \pm 1.67$ & $3.85 \pm 1.13$ & 0.181 & 0.738 & 0.041 \\
\hline & $\mathrm{D}_{\text {mean }}(\mathrm{Gy})$ & $1.09 \pm 0.97$ & $0.65 \pm 0.42$ & $1.74 \pm 0.40$ & 0.070 & 0.161 & 0.003 \\
\hline
\end{tabular}

Table 4. The EAR of the organs with three treatment modalities(means \pm SD)

\begin{tabular}{|c|c|c|c|c|c|c|}
\hline \multirow[b]{2}{*}{ EAR } & \multirow[b]{2}{*}{ full IMRT } & \multirow[b]{2}{*}{ hybrid IMRT } & \multirow[b]{2}{*}{ hybrid VMAT } & \multicolumn{3}{|c|}{$p$-Value } \\
\hline & & & & $\begin{array}{l}\text { full IMRT vs. } \\
\text { hybrid IMRT }\end{array}$ & $\begin{array}{l}\text { full IMRT vs. } \\
\text { hybrid VMAT }\end{array}$ & $\begin{array}{c}\text { hybrid IMRT vs. } \\
\text { hybrid VMAT }\end{array}$ \\
\hline Contralateral breast & $5.139 \pm 3.568$ & $4.687 \pm 3.353$ & $7.037 \pm 3.905$ & 0.492 & 0.033 & 0.020 \\
\hline Contralateral lung & $9.079 \pm 7.911$ & $4.980 \pm 4.167$ & $18.161 \pm 5.143$ & 0.030 & 0.026 & 0.000 \\
\hline Ipsilateral lung & $99.864 \pm 25.382$ & $85.191 \pm 32.877$ & $106.410 \pm 16.332$ & 0.423 & 0.412 & 0.146 \\
\hline Esophagus & $1.029 \pm 0.752$ & $0.571 \pm 0.364$ & $1.490 \pm 0.321$ & 0.028 & 0.217 & 0.003 \\
\hline
\end{tabular}




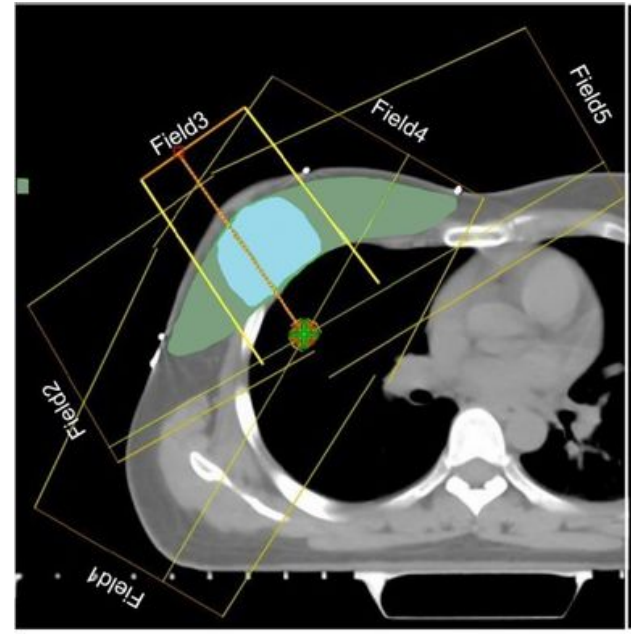

(a) Full IMRT

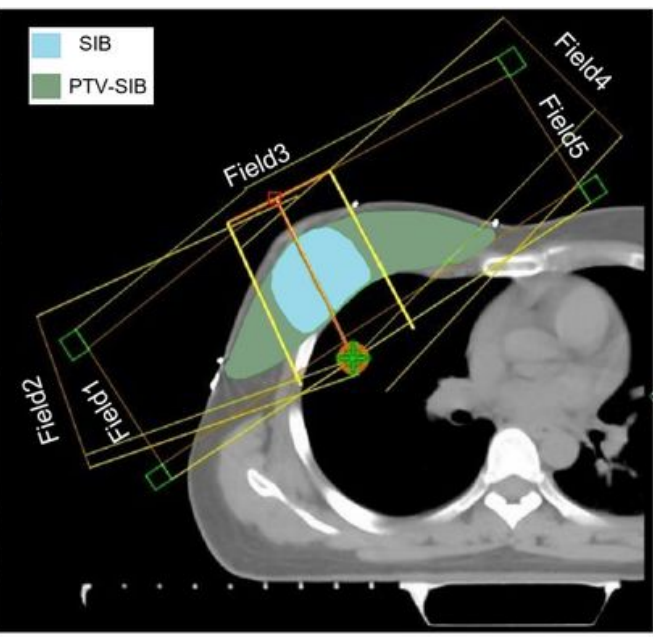

(b) Hybrid IMRT

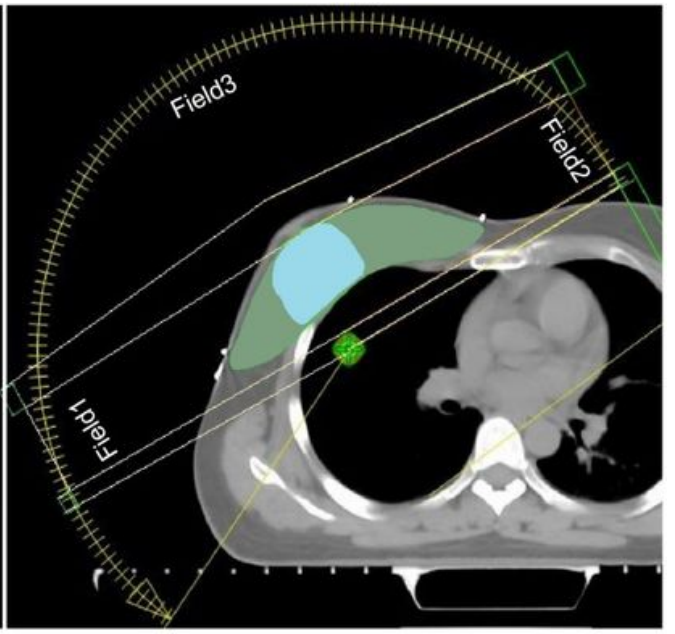

(c) Hybrid VMAT

\section{Figure 1}

Fields distributions for (a) Full IMRT, (b) hybrid IMRT and (c) Hybrid VMAT.

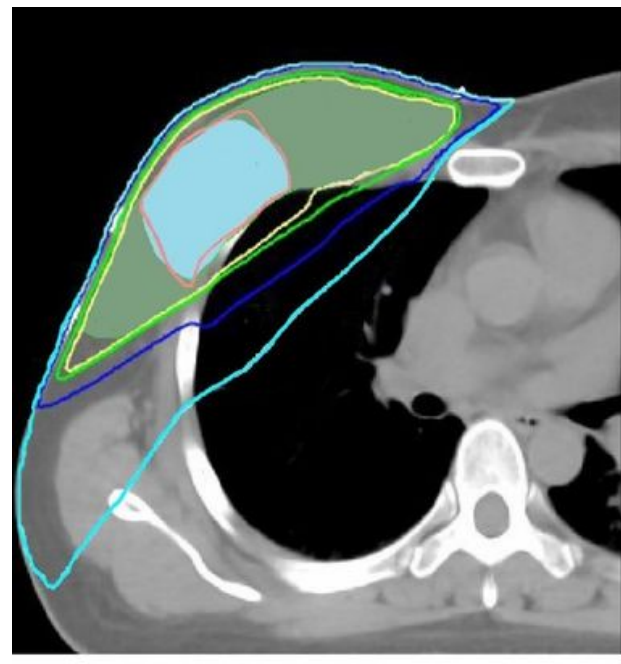

(a) Full IMRT

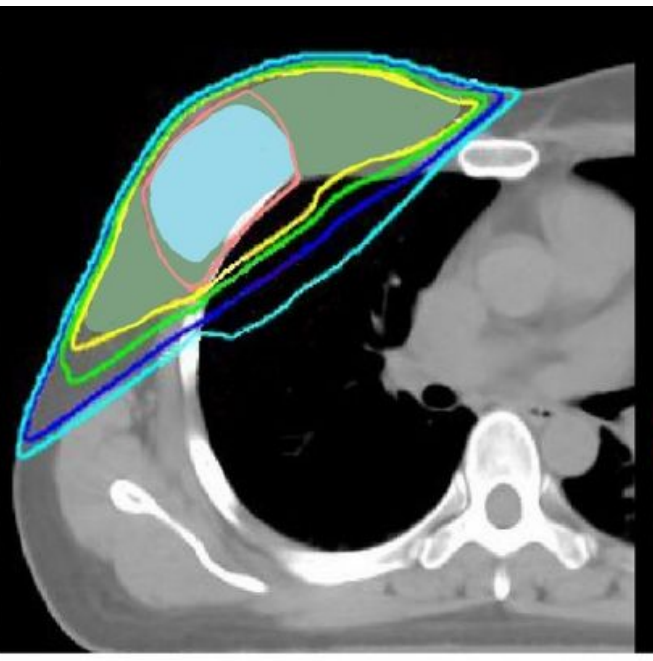

(b) Hybrid IMRT

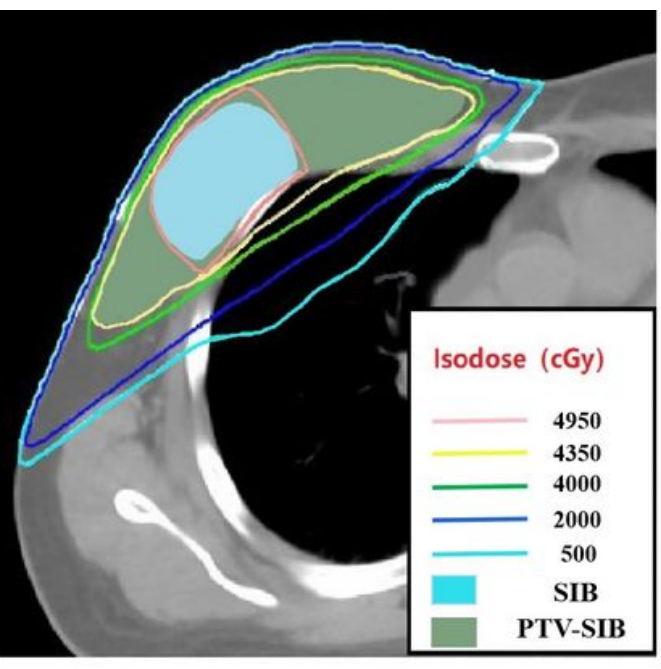

(c) Hybrid VMAT

Figure 2

Contouring of targets and comparison of planar dose distribution for one selected case. (a), (b),(c) are planar dose distribution of Full IMRT, Hybrid IMRT and Hybrid VMAT plans, respectively. 

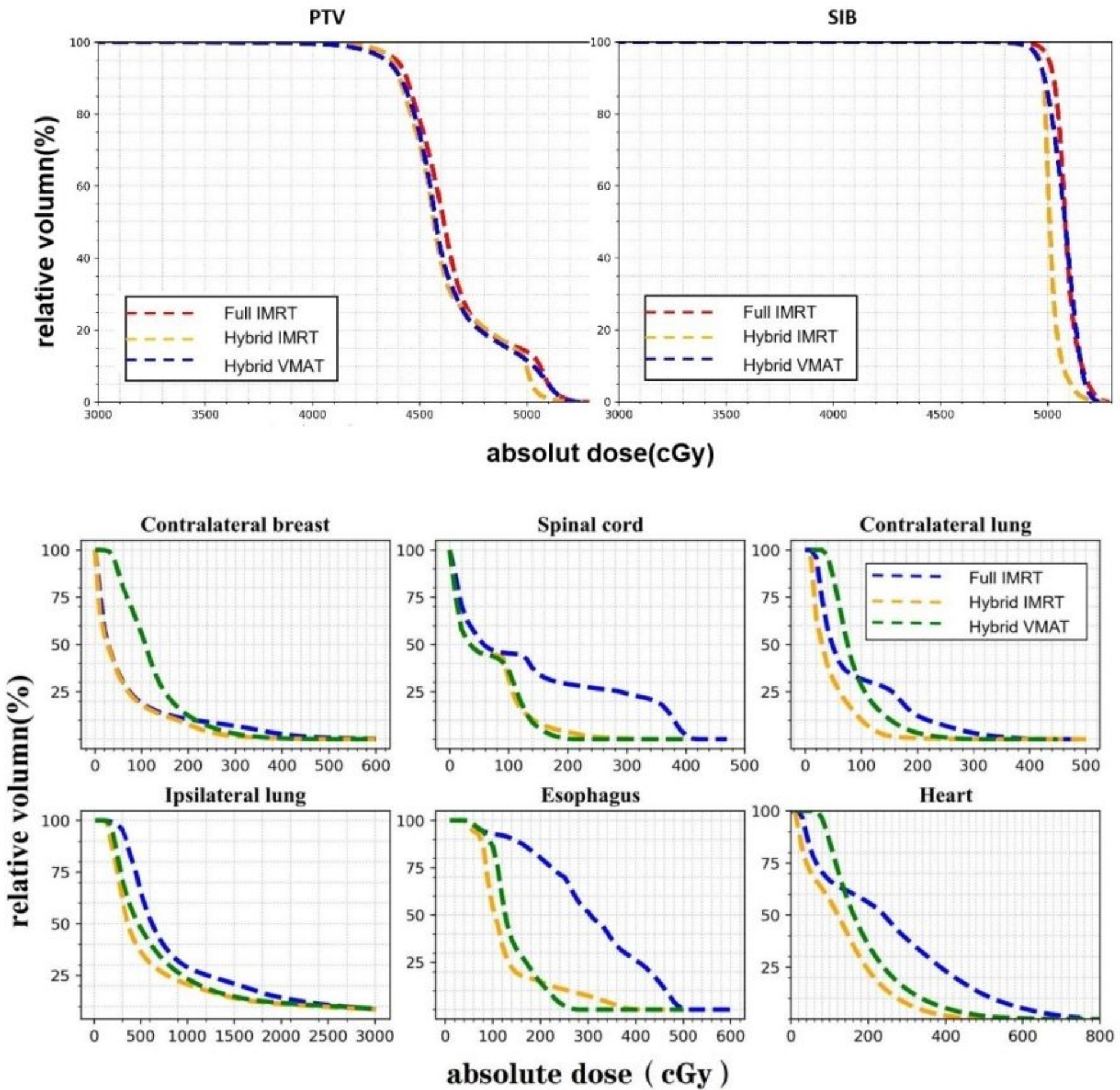

absolute dose (cGy )

Fig. 3 Representative DVHs for targets and OARs

\section{Figure 3}

The DVHs curves of the targets with prescription dose of $43.5 \mathrm{~Gy} / 15 \mathrm{~F}$ and $49.5 \mathrm{~Gy} / 15 \mathrm{~F}$ respectively (upper rows) and OARs (lower rows) for Full IMRT plan, Hybrid IMRT plan and Hybrid VMAT plan. 